ULB-TH-99/27

\title{
Refining the anomaly consistency condition
}

\author{
Glenn Barnich \\ Physique Théorique et Mathématique, Université Libre de Bruxelles, \\ Campus Plaine C.P. 231, B-1050 Bruxelles, Belgium
}

\begin{abstract}
In the extended antifield formalism, a quantum BRST differential for anomalous gauge theories is constructed. Local BRST cohomological classes are characterized, besides the form degree and the ghost number, by the length of their descents and of their lifts, and this both in the standard and the extended antifield formalism. It is shown that during the BRST invariant renormalization of a local BRST cohomological class, the anomaly that can appear is constrained to be a local BRST cohomological class with a shorter descent and a longer lift than the given class. As an application of both results, a simple approach to the Adler-Bardeen theorem for the non abelian gauge anomaly is proposed. It applies independently of the gauge fixing, of power counting restrictions and does not rely on the use of the Callan-Symanzik equation.
\end{abstract}

11.10.Gh, 11.15.Bt

Typeset using REVTEX 


\section{INTRODUCTION}

Upon quantization, symmetries of the classical action can be affected by anomalies. These anomalies have been shown to satisfy important consistency conditions following from the algebra of the symmetries [1]. The consistency conditions have been elegantly reformulated as a cohomological problem, first in the case of the gauge anomaly in YangMills theories [2, 4] and then also in the case of global symmetries with a closed algebra [5.6].

In the case of gauge theories, the formalism has been generalized some time ago [7],8] to cover generic gauge theories, and the expression of the anomaly consistency in that context has been discussed 9, 10]. Only recently, the corresponding generalization in the case of global symmetries with a generic algebra has been achieved [11].

In the same way, one can study the constraints on the anomalies to an invariant renormalization of symmetric integrated and non integrated operators [12]. In the cohomological reformulation, symmetric integrated or non integrated operators correspond to BRST cohomological classes in ghost number 0 in the space of local functionals, respectively in the space of local functions. The non trivial anomalies that can appear can be shown to belong to the corresponding BRST cohomological classes in ghost number 1.

The computation in the space of local functionals of the cohomology of the standard BRST differential $s$ with antifields can be reduced to the computation of a relative cohomological group in the space of local $n$-forms by introducing the space-time exterior derivative $d$. It is then related to the cohomology of $s$ in the space of form valued local functions through descent equations [13 16]. The same holds for the BRST differential $\bar{s}$ associated to the extended antifield formalism.

More generally, we will call the relative cohomological groups $H^{g, p}(s \mid d)$ and $H^{g, p}(\bar{s} \mid d)$ in ghost number $g$ and form degree $p$ local BRST cohomological groups. A more detailed analysis of the descent equations [17] shows that these groups are characterized by two integers, the length $d$ of their descents and the length $l$ of their lifts.

The purpose of this article is twofold. First, it is shown that in the extended antifield formalism, a quantum BRST differential can be constructed even for anomalous theories, because the quantum version in terms of the effective action of the extended master equation can be written as a functional differential equation. Then it is shown that both in the extended antifield formalism and in the standard antifield formalism, the anomaly appearing in the renormalization of a local BRST cohomological class with a descent of length $d$ and a lift of length $l$ are characterized by a descent which is shorter or equal to $d$ and a lift which is longer or equal to $l$.

As an application, it is shown that the anomalous master equation for Yang-Mills theories discussed in 18 20 can be viewed as a particular case of the anomalous master equation of the extended antifield formalism. Then, we discuss how the Adler-Bardeen theorem for the

non abelian gauge anomaly [21,18,22, 24] can be understood as a direct consequence of the fact that the length of the descent of the gauge anomaly is 4 , while the length of the descent of all the other cohomological classes coupled to the action is 0 . These considerations are purely cohomological, so that they do not depend on the way the gauge is fixed or on power counting restrictions. Furthermore, this aproach to the Adler-Bardeen theorem does not require the use of the Callan-Symanzik equation or assumptions on the beta functions of 
the theory.

The article is organized as follows. In the next section, we review the relevant features of the extended antifield formalism, with special care devoted to the derivation of the quantum BRST differential in the anomalous case. The characterization of local BRST cohomological groups according to the lengths of their descents and their lifts is explained in section III]. The fourth section contains the main result on the lengths of the descents and the lifts of the anomalies. An alternative derivation of the main results clarifying the underlying mechanism is presented in section $\mathrm{\nabla}$, where differentials controling the one loop anomalies arising in the renormalization of BRST cohomological groups are introduced. Anomalous Yang-Mills theory and the Adler-Bardeen theorem are discussed in section $\nabla \mathbb{1}$.

\section{THE EXTENDED ANTIFIELD FORMALISM}

\section{A. Classical theory}

The Batalin-Vilkovisky formalism [7,8] allows to formulate the BRST differential controling the gauge symmetries under renormalization for generic gauge theories. The formalism can be extended so as to include (non linear) global symmetries with a generic algebra [11]. This is achieved by coupling the BRST cohomological classes in negative ghost numbers with constant ghosts. There is a further extension to include the BRST cohomological classes in all the ghost numbers [25], which allows to take into account in a systematic way all higher order cohomological constraints due to the antibracket maps 26.

The completely extended formalism is obtained by first computing a basis for the local BRST cohomological classes $H^{g, n}(s \mid d)$ f associated to the standard differential $s$ and coupling those classes that are not already contained in the solution of the standard master equation with the help of new independent coupling constants. This action can then be extended by terms of higher orders in the new couplings in such a way that, if we denote by $\xi^{A}$ all the couplings corresponding to the independent BRST cohomological classes, the resulting action $S$ satisfies the extended master equation

$$
\frac{1}{2}(S, S)+\Delta_{c} S=0
$$

The BRST differential associated to the solution of the extended master equation is

$$
\bar{s}=(S, \cdot)+\Delta_{c}^{L},
$$

where $\Delta_{c}=\frac{\partial^{R}}{\partial \xi^{A}} f^{A}$, while $\Delta_{c}^{L}=(-)^{A} f^{A} \frac{\partial^{L}}{\partial \xi^{A}}$, with $f^{A}$ depending at least quadratically on the couplings $\xi$ alone. Both derivations satisfy $\left(\Delta_{c}\right)^{2}=0=\left(\Delta_{c}^{L}\right)^{2}$. Since there is no dependence on the fields and the antifields, $\Delta_{c}^{L}(A, B)=\left(\Delta_{c}^{L} A, B\right)+(-)^{A+1}\left(A, \Delta_{c}^{L} B\right)$, with the appropriate version holding for the right differential $\Delta_{c}$.

\footnotetext{
${ }^{1}$ We consider the local BRST cohomology in the space of Lorentz-invariant polynomials or formal power seris in the $d x^{\mu}$, the coupling constants, the fields, antifields and their derivatives.
} 
Besides the information on the invariance of the original action under the non trivial gauge symmetries and their commutator algebra contained in the standard solution of the master equation, the extended master equation contains the information on the antibracket algebra of all local BRST cohomological classes associated to the standard BRST differential $s$. These classes contain the generators of all the generalized non trivial symmetries f of the theory in negative ghost number. This is the reason why the extended master equation encodes in particular the invariance of the original action under all the non trivial global symmetries as well as their commutator algebra. In ghost number zero, the local BRST cohomological classes $H^{g, n}(s \mid d)$ contain all the non trivial generalized observables of the theory? In ghost number 1 , these classes contain all the anomaly candidates that could affect the standard Batalin-Vilkovisky master equation and in ghost number strictly greater than 1 , they contain the anomaly candidates in ghost number $g+1$ that could occur because the anomaly candidates in ghost number $g$ have been coupled to the action.

The cohomology of BRST differential $\bar{s}$ of the extended formalism in the space $F$ of $\xi$ dependent local functionals in the fields, the antifields and their derivatives, is isomorphic to the cohomology of

$$
s_{\Delta_{c}}=\left[\Delta_{c}, \cdot\right]
$$

in the space of graded right derivations $\lambda=\frac{\partial^{R} .}{\partial \xi^{A}} \lambda^{A}$, with $\lambda^{A}$ a function of $\xi$ alone, $[\cdot, \cdot]$ being the graded commutator for graded right derivations. If $\mu$ is a $s_{\Delta_{c}}$ cocycle, the corresponding $\bar{s}$ cocycle is given by $\mu S=\frac{\partial^{R} S}{\partial \xi^{B}} \mu^{B}$. In particular, the general solution to the system of equations

$$
\left\{\begin{array}{c}
\mu S+\bar{s} C=0, \\
s_{\Delta_{c}} \mu=0
\end{array}\right.
$$

is given by

$$
\left\{\begin{array}{c}
C=\bar{s} D+\nu S \\
\mu=s_{\Delta_{c}} \nu
\end{array}\right.
$$

\section{B. Quantum theory}

In the usual version of the BRST-Zinn-Justin-Batalin-Vilkovisky set-up, there are two main issues to be considered (see e.g. [28,29]): stability and anomalies.

\footnotetext{
${ }^{2}$ Higher order symmetries have to be taken into account in the generic case [11].

${ }^{3}$ They correspond to local functionals built out of the original fields and their derivatives that are gauge invariant when the gauge covariant equations of motion hold, and that are linearily independent even when the gauge covariant equations of motion hold (see e.g. 27]).
} 


\section{Stability}

The problem of stability is the question if to every local BRST cohomological class $H^{0, n}(s \mid d)$ in ghost number 0 , there corresponds an independent coupling of the standard master equation. The extended formalism solves this problem by construction, because all standard cohomological classes have been coupled with independent couplings. Indeed, in the extended formalism, the differential controling the "instabilities", i.e., the divergencies and/or counterterms, is the differential $\bar{s}$, and according to the previous section, $\bar{s} A=0$ implies $A=\mu S+\bar{s} B$, where $\mu$ belongs to $H\left(s_{\Delta_{c}}\right)$, so that the non trivial part of $A$ can indeed be absorbed by a modification of the couplings of the extended master equation (see 25] for more details).

In different words, the extended antifield formalism guarantees "renormalizability in the modern sense" [30] for all gauge theories. Of course, it will be often convenient not to couple all the local BRST cohomological classes but only a subset needed to guarantee that the theory is stable.

\section{Anomalous Zinn-Justin equation}

In the standard set-up, the question of anomalies is mostly reduced to the computation of the local BRST cohomological group $H^{1, n}(s \mid d)$ in ghost number 1 and to a discussion of the coefficients of the corresponding classes. In the presence of anomalies, there is no differential on the quantum level associated to the anomalously broken Zinn-Justin equation for the effective action. In the extended antifield formalism however, because all the local BRST cohomological classes in positive ghost numbers have been coupled to the solution of the master equation, the broken Zinn-Justin equation can be written as a functional differential equation and an associated differential exists, even in the presence of anomalies. To show this, is the object of the remainder of this subsection t

The quantum action principle 32 34 applied to (2.1) gives

$$
\frac{1}{2}(\Gamma, \Gamma)+\Delta_{c} \Gamma=\hbar \mathcal{A} \circ \Gamma
$$

where $\Gamma$ is the renormalized generating functional for 1PI vertices associated to the solution $S$ of the extended master equation and the local functional $\mathcal{A}$ is an element of $F$ in ghost number 1. Applying $(\Gamma, \cdot)+\Delta_{c}^{L}$ to $(2.6)$, the l.h.s vanishes identically because of the graded Jacobi identity for the antibracket and the properties of $\Delta_{c}$, so that one gets the consistency condition $(\Gamma, \mathcal{A} \circ \Gamma)+\Delta_{c}^{L} \mathcal{A} \circ \Gamma=0$. To lowest order in $\hbar$, this gives $\bar{s} \mathcal{A}=0$, the general solution of which can be writen as

\footnotetext{
${ }^{4}$ We rederive section 4 of [31] in a more appropriate notation, insisting on the existence of the quantum BRST differential in the anomalous case and its relation to its classical counterpart $\bar{s}$. Note that the relation after (4.9) of that paper should read $s_{Q} \frac{\partial^{R}}{\partial \xi^{A}} \sigma^{A}=\frac{1}{2} \frac{\partial^{R}}{\partial \xi^{A}}[\sigma, \sigma]^{A}$ instead of $s_{Q} \frac{\partial^{R} \cdot \dot{\partial} \xi^{A}}{A}=0$.
} 


$$
\mathcal{A}=-\frac{\partial^{R} S}{\partial \xi^{A}} \Delta_{1}^{A}+\bar{s} \Sigma_{1}
$$

with $\left[\Delta_{c}, \Delta_{1}\right]=0$, because of the relation between the $\bar{s}$ and the $s_{\Delta_{c}}$ cohomologies discussed in the previous subsection.

If one now defines $S^{1}=S-\hbar \Sigma_{1}$, the corresponding generating functional admits the expansion $\Gamma^{1}=\Gamma-\hbar \Sigma_{1}+O\left(\hbar^{2}\right)$ and satisfies $\frac{1}{2}\left(\Gamma^{1}, \Gamma^{1}\right)+\Delta^{1} \Gamma^{1}=O\left(\hbar^{2}\right)$, where $\Delta^{1}=\Delta_{c}+\hbar \Delta_{1}$. On the other hand, the quantum action principle applied to $\frac{1}{2}\left(S^{1}, S^{1}\right)+\Delta^{1} S^{1}=O(\hbar)$ implies $\frac{1}{2}\left(\Gamma^{1}, \Gamma^{1}\right)+\Delta^{1} \Gamma^{1}=\hbar \overline{\mathcal{A}} \circ \Gamma^{1}$, for a local functional $\overline{\mathcal{A}}$. Comparing the two expressions, we deduce that

$$
\frac{1}{2}\left(\Gamma^{1}, \Gamma^{1}\right)+\Delta^{1} \Gamma^{1}=\hbar^{2} \mathcal{A}^{\prime} \circ \Gamma^{1},
$$

for a local functional $\mathcal{A}^{\prime}$. Applying now $\left(\Gamma^{1}, \cdot\right)+\left(\Delta^{1}\right)^{L}$, one gets as consistency condition

$$
\frac{1}{2}\left[\Delta^{1}, \Delta^{1}\right] \Gamma^{1}+\hbar^{2}\left(\left(\Gamma^{1}, \mathcal{A}^{\prime} \circ \Gamma^{1}\right)+\Delta^{1} \mathcal{A}^{\prime} \circ \Gamma^{1}\right)=0,
$$

giving to lowest order

$$
1 / 2\left[\Delta_{1}, \Delta_{1}\right] S+\bar{s} \mathcal{A}^{\prime}=0 .
$$

Since $1 / 2\left[\Delta_{1}, \Delta_{1}\right]$ is a $s_{\Delta_{c}}$ cocycle because of the graded Jacobi identity for the graded commutator, equations (2.4) and (2.5) of the previous section imply that the general solution to this equation is

$$
\begin{array}{r}
\frac{1}{2}\left[\Delta_{1}, \Delta_{1}\right]+\left[\Delta_{c}, \Delta_{2}\right]=0 \\
\mathcal{A}^{\prime}=\bar{s} \Sigma_{2}-\frac{\partial^{R} S}{\partial \xi^{B}} \Delta_{2}^{B} .
\end{array}
$$

The redefinition $S^{2}=S^{1}-\hbar^{2} \Sigma_{2}$ then allows to achieve

$$
\frac{1}{2}\left(\Gamma^{2}, \Gamma^{2}\right)+\Delta^{2} \Gamma^{2}=\hbar^{3} \mathcal{A}^{\prime \prime} \circ \Gamma^{2},
$$

for a local functional $\mathcal{A}^{\prime \prime}$, with $\Delta^{2}=\Delta^{1}+\hbar^{2} \Delta_{2}$. The reasoning can be pushed recursively to all orders with the result

$$
\frac{1}{2}\left(\Gamma^{\infty}, \Gamma^{\infty}\right)+\Delta^{\infty} \Gamma^{\infty}=0
$$

where $\Gamma^{\infty}$ is associated to the action $S^{\infty}=S-\Sigma_{k=1} \hbar^{k} \Sigma_{k}$ and $\Delta^{\infty}=\Delta_{c}+\hbar \Delta_{1}+\hbar^{2} \Delta_{2}+\ldots$ satisfies $\left(\Delta^{\infty}\right)^{2}=0$. The associated quantum BRST differential is

$$
s^{q}=\left(\Gamma^{\infty}, \cdot\right)+\left(\Delta^{\infty}\right)^{L} .
$$

In the limit $\hbar$ going to zero, it coincides with the classical differential $\bar{s}$.

In the extended antifield formalism, the anomalous Zinn-Justin equation can thus be written as a functional differential equation for the renormalized effective action. The derivations $\Delta_{1}, \Delta_{2}, \ldots$ are guaranteed to exist due to the quantum action principles. They satisfy a priori cohomological restrictions due to the fact that the differential $\Delta^{\infty}$ is a formal deformation with deformation parameter $\hbar$ of the differential $\Delta_{c}$. In the context of chiral Yang-Mills theories, where $\Delta_{c}=0$, an anomalous master equation of the form (2.14) for the renormalized effective action has appeared for the first time in [18]. 


\section{Renormalization of local BRST cohomological classes of the extended formalism}

Associated quantum extension of the classical BRST cohomological classes $\lambda_{0} S$ of the extended formalism are given by $\lambda_{0} \Gamma^{\infty}$. By acting with $\lambda_{0}$ on (2.14), one gets

$$
s^{q} \lambda_{0} \Gamma^{\infty}=-\left[\Delta^{\infty}, \lambda_{0}\right] \Gamma^{\infty} .
$$

The derivation $\left[\Delta^{\infty}, \lambda_{0}\right]=\sum_{n \geq L} \hbar^{n} \mu_{n}$ on the right hand side is of order at least $\hbar, L \geq 1$, because $\left[\Delta_{c}, \lambda_{0}\right]=0$ and corresponds to the anomaly in the invariant renormalization of $\lambda_{0} S$. The question then is whether there exists modified quantum extension $\lambda^{\infty} \Gamma^{\infty}$, with $\lambda^{\infty}=\lambda_{0}+\hbar \lambda_{1}+\ldots$, such that

$$
s^{q} \lambda^{\infty} \Gamma^{\infty}=0
$$

This is for instance the case if $\lambda_{0}$ corresponds to the trivial cohomological class, $\lambda_{0}=\left[\Delta_{c}, \nu_{0}\right]$. The searched for extension can then simply be taken to be $\lambda^{\infty}=\left[\Delta^{\infty}, \nu_{0}\right]$.

Because $\left[\Delta^{\infty},\left[\Delta^{\infty}, \lambda_{0}\right]\right]=0$, the lowest order part of the anomaly, $\mu_{L}$ is an $s_{\Delta_{c}}$ cocyle, $\left[\Delta_{c}, \mu_{L}\right]=0$. Suppose that $\mu_{L}$ is a trivial solution to this equation, $\mu_{L}=-\left[\Delta_{c}, \lambda_{L}\right]$. The modified quantum extension $\left(\lambda_{0}+\hbar^{L} \lambda_{L}\right) \Gamma^{\infty}$ allows to push the anomaly to order $L+1$.

Hence, to lowest order in $\hbar$, the non trivial part of the anomaly in the renormalization of a classical cohomological class $H^{g}\left(s_{\Delta_{c}}\right)$ is constrained to belong to $H^{g+1}\left(s_{\Delta_{c}}\right)$. All the quantum information on this anomaly is encoded in the derivations $\Delta_{1}, \Delta_{2}, \ldots$ and the whole discussion has been shifted from local functionals to derivations of functions of the coupling constants.

\section{CHARACTERIZATION OF LOCAL BRST COHOMOLOGICAL CLASSES}

In this and the following section, we decompose the space $\Omega$ of Lorentz-invariant polynomials or formal powers series in the $d x^{\mu}$, the couplings $\xi^{A}$, the fields, antifields and their derivatives into the direct sum of the constants and the remaining part, $\Omega=\mathbf{R} \oplus \Omega_{+}$. We have $\bar{s} \alpha=0=d \alpha$, for a constant $\alpha$ and $d \Omega_{+} \subset \Omega_{+}$. We furthermore assume that if $\bar{s} \omega=\alpha$ for a constant $\alpha$, then $\alpha=0$, which amounts to assuming that the equations of motions are consistent (see the discussion in chapter 9 of 35$]$ ). This means that $\bar{s} \tilde{\Omega} \subset \tilde{\Omega}$. Hence, we can consider the cohomological groups $H\left(\bar{s}, \Omega_{+}\right), H\left(\bar{s} \mid d, \Omega_{+}\right)$and $H\left(d, \Omega_{+}\right)$.

By analyzing the cohomological groups $H(\bar{s} \mid d)$ (the space $\Omega_{+}$being always understood in the following) using descent equations, one can prove [17 that the elements of these groups can be classified into chains of length $r$ with an obstruction to further lifts and chains of length $s$ whose lifts are unobstructed, i.e., chains with a non trivial element in degree $n$ (see also [36 38]; we follow here the notations of the review [35], where explicit proofs of the statements below can be found). More precisely, we have $H^{p}(d)=0, p \leq n-1$, and there exists a basis

$$
\left\{\left[h_{i_{r}}^{0}\right],\left[\hat{h}_{i_{r}}\right],\left[e_{\alpha_{s}}^{0}\right]\right\}
$$

of $H(\bar{s})$, for $r=0, \ldots, n-1, s=0, \ldots, n$, such that a corresponding basis of $H(\bar{s} \mid d)$ is given by 


$$
\left\{\left[h_{i_{r}}^{q}\right],\left[e_{\alpha_{s}}^{p}\right]\right\}
$$

for $q=0, \ldots r$ and $p=0, \ldots, s$, with

$$
\begin{gathered}
\bar{s} h_{i_{r}}^{r+1}+d h_{i_{r}}^{r}=\hat{h}_{i_{r}}, \\
\bar{s} h_{i_{r}}^{r}+d h_{i_{r}}^{r-1}=0, \\
\vdots \\
\bar{s} h_{i_{r}}^{1}+d h_{i_{r}}^{0}=0, \\
\bar{s} h_{i_{r}}^{0}=0,
\end{gathered}
$$

and

$$
\begin{gathered}
\text { form degree } e_{\alpha_{s}}^{s}=n, d e_{\alpha_{s}}^{s}=0 \\
\bar{s} e_{\alpha_{s}}^{s}+d e_{\alpha_{s}}^{s-1}=0 \\
\vdots \\
\bar{s} e_{\alpha_{s}}^{1}+d e_{\alpha_{s}}^{0}=0 \\
\bar{s} e_{\alpha_{s}}^{0}=0
\end{gathered}
$$

The cohomological group $H(\bar{s})$ can thus be decomposed into elements $\left[e_{\alpha_{s}}^{0}\right]$ that are bottoms of unobstructed chains of length $s$, elements $\left[h_{i_{r}}^{0}\right]$ that are bottoms of obstructed chains of length $r$ and obstructions $\left[\hat{h}_{i_{r}}\right]$ to chains of length $r$.

For the cohomological group $H(\bar{s} \mid d)$, the element $\left[h_{i_{r}}^{l}\right]$ is said to be the element of level $l$ of a chain of length $r$ with obstruction; it has $l$ non trivial descents and $r-l$ non trivial lifts ; while the element $\left[e_{\alpha_{s}}^{l}\right]$ is said to be the element of level $l$ of a chain of length $s$ without obstructions, it has $l$ non trivial descents and $s-l$ non trivial lifts.

One can furthermore show that the general solution to a set of descent equations involving at most $l$ steps, $\bar{s} \omega^{l}+d \omega^{l-1}=0, \bar{s} \omega^{l-1}+d \omega^{l-2}=0, \ldots, \bar{s} \omega^{0}=0$, can be written in terms of the above elements as

$$
\omega^{l}=\sum_{q=0}^{l} \sum_{r=l-q}^{n-1} \lambda_{q}^{i_{r}} h_{i_{r}}^{l-q}+\sum_{p=0}^{l} \sum_{s=l-p}^{n} \mu_{p}^{\alpha_{s}} e_{\alpha_{s}}^{l-p}+\bar{s} \eta^{l}+d\left[\eta^{l-1}+\sum_{r=0}^{n-1} \nu^{(l) i_{r}} h_{i_{r}}^{r}\right],
$$

with $\eta^{-1}=0$. This means that a $\bar{s}$ modulo $d$ cocycle which has $l$ non trivial descents, is a linear combination of all elements of the chains (3.3) and (3.4) which have $l$ or less non trivial descents. If such a linear combination is $\bar{s}$ modulo $d$ trivial, the coefficients of the linear combination must vanish, i.e.,

$$
\sum_{q=0}^{l} \sum_{r=l-q}^{n-1} \lambda_{q}^{i_{r}} h_{i_{r}}^{l-q}+\sum_{p=0}^{l} \sum_{s=l-p}^{n} \mu_{p}^{\alpha_{s}} e_{\alpha_{s}}^{l-p}=\bar{s}()+d() .
$$

implies that $\lambda_{q}^{i_{r}}=0=\mu_{p}^{\alpha_{s}}$.

\section{LENGTHS OF DESCENT AND LIFTS OF LOWEST ORDER ANOMALIES}

Let us now investigate the anomalies in the BRST invariant renormalization of a chain (3.4) of length $s$ without obstructions. We follow the approach of [39], which consists in 
considering simultaneously the anomalies for a whole chain of descent equations (for a review, see [29]). Using the quantum action principles, one can prove (as in lemma 1 in the appendix of [31]) that if $a$ is a local form, then

$$
\bar{s} a \circ \Gamma^{\infty}=s^{q} a \circ \Gamma^{\infty}+\hbar b \circ \Gamma^{\infty},
$$

for some local form $b$. When applied to the chain (3.4), we find that the quantum version of this chain is

$$
\begin{gathered}
\text { form degree } e_{\alpha_{s}}^{s} \circ \Gamma^{\infty}=n, d e_{\alpha_{s}}^{s} \circ \Gamma^{\infty}=0, \\
s^{q} e_{\alpha_{s}}^{s} \circ \Gamma^{\infty}+d e_{\alpha_{s}}^{s-1} \circ \Gamma^{\infty}=\hbar a_{\alpha_{s}}^{s} \circ \Gamma^{\infty}, \\
\vdots \\
s^{q} e_{\alpha_{s}}^{1} \circ \Gamma^{\infty}+d e_{\alpha_{s}}^{0} \circ \Gamma^{\infty}=\hbar a_{\alpha_{s}}^{1} \circ \Gamma^{\infty}, \\
s^{q} e_{\alpha_{s}}^{0} \circ \Gamma^{\infty}=\hbar a_{\alpha_{s}}^{0} \circ \Gamma^{\infty},
\end{gathered}
$$

where $a_{\alpha_{s}}^{l} \circ \Gamma^{\infty}=a_{\alpha_{s}}^{s}+O(\hbar)$ for a local function $a_{\alpha_{s}}^{l}$. Applying $s^{q}$, we get the consistency condition,

$$
\begin{gathered}
\text { form degree } a_{\alpha_{s}}^{s} \circ \Gamma^{\infty}=n, d a_{\alpha_{s}}^{s} \circ \Gamma^{\infty}=0 \\
\qquad \begin{array}{c}
s^{q} a_{\alpha_{s}}^{s} \circ \Gamma^{\infty}+d a_{\alpha_{s}}^{s-1} \circ \Gamma^{\infty}=0, \\
\vdots \\
s^{q} a_{\alpha_{s}}^{1} \circ \Gamma^{\infty}+d a_{\alpha_{s}}^{0} \circ \Gamma^{\infty}=0, \\
s^{q} a_{\alpha_{s}}^{0} \circ \Gamma^{\infty}=0 .
\end{array}
\end{gathered}
$$

At lowest order in $\hbar$, we get

$$
\begin{gathered}
\text { form degree } a_{\alpha_{s}}^{s}=n, d a_{\alpha_{s}}^{s}=0 \\
\bar{s} a_{\alpha_{s}}^{s}+d a_{\alpha_{s}}^{s-1}=0, \\
\vdots \\
\bar{s} a_{\alpha_{s}}^{1}+d a_{\alpha_{s}}^{0}=0, \\
\bar{s} a_{\alpha_{s}}^{0}=0 .
\end{gathered}
$$

Using equation (3.5) and the fact that the form degree of $a_{\alpha_{s}}^{s}$ is $n$, it follows that

$$
a_{\alpha_{s}}^{l}=\sum_{p=0}^{l} \mu_{p}^{\beta_{s-p}} e_{\alpha_{s}}^{l-p}+\overline{\beta_{s-p}} \eta_{\alpha_{s}}^{l}+d\left[\eta_{\alpha_{s}}^{l-1}+\sum_{r \geq 0} \nu_{\alpha_{s}}^{(l) i_{r}} h_{i_{r}}^{r}\right],
$$

for $l=0, \ldots, s$. This gives our first result:

The anomaly in the renormalization of an element of level $l$ of a chain of length $s$ without obstructions involves at most elements of chains of the same type with less non trivial descents and the same number of non trivial lifts.

For the anomalies for a chain with obstruction (3.3), we get,

$$
\begin{gathered}
s^{q} h_{i_{r}}^{r+1} \circ \Gamma^{\infty}+d h_{i_{r}}^{r} \circ \Gamma^{\infty}=\hat{h}_{i_{r}} \circ \Gamma^{\infty}+\hbar a_{i_{r}}^{r+1} \circ \Gamma^{\infty}, \\
s^{q} h_{i_{r}}^{r} \circ \Gamma^{\infty}+d h_{i_{r}}^{r-1} \circ \Gamma^{\infty}=\hbar a_{i_{r}}^{r} \circ \Gamma^{\infty}, \\
\vdots \\
s^{q} h_{i_{r}}^{1} \circ \Gamma^{\infty}+d h_{i_{r}}^{0} \circ \Gamma^{\infty}=\hbar a_{i_{r}}^{1} \circ \Gamma^{\infty}, \\
s^{q} h_{i_{r}}^{0} \circ \Gamma^{\infty}=\hbar a_{i_{r}}^{0} \circ \Gamma^{\infty},
\end{gathered}
$$


We also have

$$
s^{q} \hat{h}_{i_{r}} \circ \Gamma^{\infty}=-\hbar \hat{a}_{i_{r}} \circ \Gamma^{\infty} .
$$

Applying $s^{q}$ gives $s^{q} \hat{a}_{i_{r}} \circ \Gamma^{\infty}=0$ and then to lowest order, $\bar{s} \hat{a}_{i_{r}}=0$. Applying now $s^{q}$ to the chain (4.6) gives

$$
\begin{gathered}
s^{q} a_{i_{r}}^{r+1} \circ \Gamma^{\infty}+d a_{i_{r}}^{r} \circ \Gamma^{\infty}=\hat{a}_{i_{r}} \circ \Gamma^{\infty}, \\
s^{q} a_{i_{r}}^{r} \circ \Gamma^{\infty}+d a_{i_{r}}^{r-1} \circ \Gamma^{\infty}=0, \\
\vdots \\
s^{q} a_{i_{r}}^{1} \circ \Gamma^{\infty}+d a_{i_{r}}^{1} \circ \Gamma^{\infty}=0, \\
s^{q} a_{i_{r}}^{0} \circ \Gamma^{\infty}=0,
\end{gathered}
$$

and to lowest order,

$$
\begin{gathered}
\bar{s} a_{i_{r}}^{r+1}+d a_{i_{r}}^{r}=\hat{a}_{i_{r}}, \\
\bar{s} a_{i_{r}}^{r}+d a_{i_{r}}^{r-1}=0, \\
\vdots \\
\bar{s} a_{i_{r}}^{1}+d a_{i_{r}}^{1}=0, \\
\bar{s} a_{i_{r}}^{0}=0,
\end{gathered}
$$

On the one hand, it follows from (3.5) that

$$
a_{i_{r}}^{l}=\sum_{q=0}^{l} \sum_{r^{\prime}=r-q}^{n-1} \lambda_{q}^{j_{r^{\prime}}}{ }_{i_{r}} h_{j_{r^{\prime}}}^{l-q}+\sum_{p=0}^{l} \sum_{s=r-p+1}^{n} \mu_{p}^{\beta_{s}} e_{i_{r}}^{l-p}+\bar{s} \eta_{i_{r}}^{l}+d\left[\eta_{i_{r}}^{l-1}+\sum_{r^{\prime}=0}^{n-1} \nu_{i_{r}}^{(l) j_{r^{\prime}}} h_{j_{r^{\prime}}}^{r^{\prime}}\right]
$$

for $l=0, \ldots, r$, while on the other hand, the cohomology of $\bar{s}$ implies

$$
\hat{a}_{i_{r}}=\sum_{r^{\prime}=0}^{n-1} \alpha_{i_{r}}^{j_{r^{\prime}}} \hat{h}_{j_{r^{\prime}}}+\sum_{r^{\prime}=0}^{n-1} \beta_{i_{r}}^{j_{r^{\prime}}} h_{i_{r}}^{0}+\sum_{s=0}^{n} \gamma_{i_{r}}^{\alpha_{s}} e_{\alpha_{s}}^{0}+\bar{s} \hat{o}_{i_{r}} .
$$

Applying $d$ to $(4.10)$ at $l=r$ gives

$$
\begin{aligned}
d a_{i_{r}}^{r}=-\sum_{q=0}^{r} \sum_{r^{\prime}=r-q+1}^{n-1} \lambda_{q i_{r}}^{i_{r}^{\prime}} \bar{s} h_{i_{r^{\prime}}}^{r-q+1} & -\sum_{p=0}^{r} \sum_{s=r-p+1}^{n} \mu_{p}^{\beta_{s}} \bar{s} e_{\beta_{s}}^{r-p+1}-\bar{s} d \eta_{i_{r}}^{r} \\
& +\sum_{q=0}^{r} \lambda_{q i_{r}}^{i_{r}-q}\left(-\bar{s} h_{i_{r-q}}^{r-q+1}+\hat{h}_{i_{r-q}}\right) .
\end{aligned}
$$

Injecting now (4.11) and (4.12) into the first equation of (4.9) gives first of all $\beta_{i_{r}}^{j_{r^{\prime}}}=0=\gamma_{i_{r}}^{\alpha_{s}}$ and also

$$
\hat{a}_{i_{r}}=\sum_{q=0}^{r} \lambda_{q}^{j_{r-q}} \hat{h}_{i_{r}}+\bar{s} \hat{o}_{i_{r}}
$$

and then, 


$$
\begin{array}{r}
\bar{s}\left(a_{i_{r}}^{r+1}-\sum_{q=0}^{r} \sum_{r^{\prime}=r-q+1}^{n-1} \lambda_{q_{i_{r}}}^{i_{r^{\prime}}} h_{i_{r^{\prime}}}^{r-q+1}-\sum_{p=0}^{r} \sum_{s=r-p+1}^{n} \mu_{p i_{r}}^{\beta_{s}} e_{\beta_{s}}^{r-p+1}\right. \\
\left.-\sum_{q=0}^{r} \lambda_{q_{i_{r}} i_{i_{r}-q}}^{i_{r-q}} h_{i_{i_{r}}-q+1}^{r-1}-d \hat{o}_{i_{r}}\right)=0
\end{array}
$$

so that, using the cohomology of $\bar{s}$,

$$
\begin{array}{r}
a_{i_{r}}^{r+1}=\sum_{q=0}^{r+1} \sum_{r^{\prime}=r-q}^{n-1} \lambda_{q}^{i_{r^{\prime}}}{ }_{i_{r}} h_{i_{r^{\prime}}}^{r-q+1}+\sum_{p=0}^{r+1} \sum_{s=r-p+1}^{n} \mu_{p}^{\beta_{s}}{ }_{i_{r}} e_{\beta_{s}}^{r-p+1} \\
+d\left[\eta_{i_{r}}^{r}+\sum_{r^{\prime}=0}^{n-1} \nu_{i_{r}}^{(r+1) j_{r^{\prime}}} h_{j_{r^{\prime}}}^{r^{\prime}}\right]+\hat{o}_{i_{r}}+\bar{s} \eta_{i_{r}}^{r+1} .
\end{array}
$$

Our second result is then:

The anomaly in the renormalization of an element of level $l$ of a chain of length $r$ with obstructions involves at most elements of chains with obstructions with less non trivial descents and more non trivial lifts and elements of chains without obstructions with less non trivial descents and strictly more non trivial lifts.

Let us now rewrite (4.5) and (4.10) at $l=0$ as

$$
\begin{array}{r}
a_{\alpha_{s}}^{0}=\mu_{0}^{\beta_{s} \alpha_{s}} e_{\beta_{s}}^{0}+\bar{s}\left[\eta_{\alpha_{s}}^{0}-\sum_{r=0}^{n-1} \nu_{\alpha_{s}}^{(0) i_{r}} h_{i_{r}}^{r+1}\right]+\sum_{r=0}^{n-1} \nu_{\alpha_{s}}^{(0) i_{r}} \hat{h}_{i_{r}} \\
a_{i_{r}}^{0}=\sum_{r^{\prime}=r}^{n-1} \lambda_{0}^{j_{r^{\prime}}{ }_{i_{r}}} h_{j_{r^{\prime}}}^{0}+\sum_{s=r+1}^{n} \mu_{0}^{\beta_{s}} e_{i_{r}}^{0} e_{\beta_{s}}^{0}+\bar{s}\left[\eta_{i_{r}}^{0}-\sum_{r^{\prime}=0}^{n-1} \nu_{i_{r}}^{(0) j_{r^{\prime}}} h_{j_{r^{\prime}}}^{r^{\prime}+1}\right]+\sum_{r^{\prime}=0}^{n-1} \nu_{i_{r}}^{(0) j_{r^{\prime}}} \hat{h}_{j_{r^{\prime}}}
\end{array}
$$

Combined with (4.13), our third result on anomalies in the renormalization of elements of $H(\bar{s})$ is accordingly:

The anomaly in the renormalization of obstructions to chains of length $r$ involves at most obstructions to shorther chains; the anomaly in the renormalization of bottoms of unobstructed chains of length s involves at most bottoms of unobstructed chains of the same length and obstructions to chains of all possible lengths; the anomaly in the renormalization of bottoms of obstructed chains of length $r$ involves at most bottoms of obstructed chains of greater length, bottoms of unobstructed chains of strictly greater length and obstructions to chains of all possible lengths.

\section{DIFFERENTIALS ASSOCIATED TO ONE LOOP ANOMALIES}

A different, more compact, way to formulate and prove the results of section III and IV is to use the exact couple describing the descent and the associated spectral sequence [17].

Indeed, the diagram

$$
\begin{gathered}
H(\bar{s} \mid d) \stackrel{\mathcal{D}}{\longrightarrow} H(\bar{s} \mid d) \\
i_{0} \nwarrow \swarrow l_{0} \\
H(\bar{s})
\end{gathered}
$$


can be shown to be exact at all corners. The various maps are defined as follows: $i_{0}$ is the map which consists in regarding an element of $H(\bar{s})$ as an element of $H(\bar{s} \mid d), i_{0}: H(\bar{s}) \longrightarrow H(\bar{s} \mid d)$, with $i_{0}[a]=[a]$. It is well defined because every $\bar{s}$ cocycle is a $\bar{s}$ cocycle modulo $d$ and every $\bar{s}$ coboundary is a $\bar{s}$ coboundary modulo $d$. The descent homomorphism $\mathcal{D}: H^{k, l}(\bar{s} \mid d) \longrightarrow$ $H^{k+1, l-1}(\bar{s} \mid d)$ with $\mathcal{D}[a]=[b]$, if $\bar{s} a+d b=0$ is well defined because of the triviality of the cohomology of $d$ in form degree $p \leq n-1$. Finally, the map $l_{0}: H^{k+1, l-1}(\bar{s} \mid d) \longrightarrow H^{k+1, l}(\bar{s})$ is defined by $l_{0}[a]=[d a]$. It is well defined because the relation $\{\bar{s}, d\}=0$ implies that it maps cocycles to cocycles and coboundaries to coboundaries.

Associated to such an exact couple $\left(H(\bar{s} \mid d), K_{0}=H(\bar{s})\right)$, one can associate in a standard way derived exact couples $\left(\mathcal{D}^{r} H(\bar{s} \mid d), K_{r}\right)$,

$$
\begin{gathered}
\mathcal{D}^{r} H(\bar{s} \mid d) \stackrel{\mathcal{D}}{\longrightarrow} \mathcal{D}^{r} H(\bar{s} \mid d) \\
i_{r} \nwarrow^{\swarrow} l_{r} \\
K_{r},
\end{gathered}
$$

and a spectral sequence $K_{r+1}=H\left(d_{r}, K_{r}\right)$, with $K_{0} \equiv H(\bar{s})$. The maps of these exact couples are defined recursively as follows: the map $d_{r-1}=l_{r-1} \circ i_{r-1}$ can be shown to be a differential, the map $i_{r}$ is the map induced by $i_{r-1}$ in $K_{r}$, while $l_{r} \mathcal{D}[a]=l_{r-1}[a]$.

Explicitly, the differential $d_{0}: K_{0} \longrightarrow K_{0}$ is defined by $d_{0}[a]=[d a]$, where $\bar{s} a=0$. An element $k_{r} \in K_{r}$ is identified with the equivalence class $[a]_{r}$ of an element $[a] \in H(\bar{s})$, where $[a] \sim_{r}\left[a^{\prime}\right]$ if $[a]-\left[a^{\prime}\right] \in \oplus_{q=0}^{r-1} \mathrm{im} d_{q}$. The relations $d_{q} k_{r}=0, q=0, \ldots, r-1$ mean that $k_{r}$ is a bottom that can be lifted at least $r$ times, i.e., there exist $c_{1}, \ldots, c_{r+1}$ such that $\bar{s} a=0, d a+\bar{s} c_{1}=0, \ldots, d c_{r-1}+\bar{s} c_{r}=0$. Then, the differential $d_{r}$ is defined by $d_{r} k_{r}=\left[d c_{r}\right]_{r}$.

Because there are no forms of form degree higher than $n, \mathcal{D}^{n+1} H(\bar{s} \mid d)=0$ and $d_{n} \equiv 0$ so that the construction stops at $r=n$.

The space of local forms $\Omega$ is decomposed as $\Omega=E_{0} \oplus G \oplus \bar{s} G \oplus \mathbf{R}$, with $E_{0} \simeq K_{0}=H(\bar{s})$. If we define $E_{r}, F_{r-1} \subset E_{r-1}$ through $E_{r-1}=E_{r-1} \oplus E_{r} \oplus d_{r-1} F_{r-1}$ with $E_{r} \simeq K_{r}$, we get the decomposition

$$
E_{0}=F_{0} \oplus \ldots \oplus F_{n-1} \oplus E_{n} \oplus d_{r-1} F_{r-1} \oplus \ldots \oplus d_{0} F_{0}
$$

The $e_{\alpha_{s}}^{0}$ are elements of a basis of $E_{n}$ that can be lifted $s$ times before hitting form degree $n$, i.e., that are of form degree $n-s$, while $\hat{h}_{i_{r}}$ and $h_{i_{r}}^{0}$ are elements of a basis of $d_{r} F_{r}$ and $F_{r}$ respectively. This sums up the results of section [II.

Let us now define the linear map

$$
\begin{array}{r}
\delta_{0}: H^{g}(\bar{s}) \longrightarrow H^{g+1}(\bar{s}), \\
\delta_{0}[a]=[b], \text { where } s^{q} a \circ \Gamma^{\infty}=\hbar b \circ \Gamma^{\infty} .
\end{array}
$$

The map associates to a given BRST cohomological class the non trivial order $\hbar$, i.e., 1 loop contribution of its anomaly.

The map is well defined, because the consistency condition implies that $\bar{s} b=0$, and if $a=\bar{s} c, a \circ \Gamma^{\infty}=s^{q} c \circ \Gamma^{\infty}+\hbar d \circ \Gamma^{\infty}$, so that $s^{q} a \circ \Gamma^{\infty}=\hbar s^{q} d \circ \Gamma^{\infty}$, meaning that $b=\bar{s} d$, so that the map does not depend on the choice of the representative. Furthermore, this map is a differential

$$
\delta_{0}^{2}=0
$$


Indeed, if $[a]=\delta_{0}[c]$, we have $a \circ \Gamma^{\infty}=\frac{1}{\hbar} s^{q} c \circ \Gamma^{\infty}$. It follows that $s^{q} a \circ \Gamma^{\infty}=0$. A BRST cohomological class which is a $\delta_{0}$-cocycle has no 1-loop anomaly, while a BRST cohomological class which is a $\delta_{0}$-coboundary is the 1-loop anomaly of some other BRST cohomological class.

We thus have two differentials in $K_{0}=H(\bar{s}), d_{0}$ introduced above and $\delta_{0}$. These differentials anticommute,

$$
\left\{d_{0}, \delta_{0}\right\}=0 .
$$

Indeed, if $s^{q} a \circ \Gamma^{\infty}=\hbar b \circ \Gamma^{\infty}, d_{0} \delta_{0}[a]=d_{0}[b]=[d b]$, while $\delta_{0} d_{0}[a]=\delta_{0}[d a]$, and $s^{q}\left(d a \circ \Gamma^{\infty}\right)=$ $s^{q} d\left(a \circ \Gamma^{\infty}+\hbar c \circ \Gamma^{\infty}\right)=-d s^{q}\left(a \circ \Gamma^{\infty}+\hbar c \circ \Gamma^{\infty}\right)=-\hbar d\left(b \circ \Gamma^{\infty}+s^{q} c \circ \Gamma^{\infty}\right)$, so that $\delta_{0}[d a]=[-d(b+\bar{s} c)]=-[d b]$.

The relation $d_{0} \delta_{0}[a]=-\delta_{0} d_{0}[a]$ means:

- if $[a]$ belongs to im $d_{0},[a]=d_{0}[b]$, then $\delta_{0}[a]=-d_{0} \delta_{0}[b]$, i.e., if $[a]$ represents an obstruction to the lift of an element $[b]$, its anomaly represents minus the obstruction to the lift of the anomaly of $[b]$,

- if $d_{0}[a]=0$, then $d_{0} \delta_{0}[a]=0$, i.e., if $[a]$ can be lifted, then so does its anomaly $\delta_{0}[a]$,

- the anomaly in a bottom $[a]$ of $K_{0}$ that cannot be lifted is minus the anomaly of the corresponding obstruction, up to elements that can be lifted.

If we organize the space $E_{0} \simeq K_{0}=H(\bar{s})$ as $E_{0}=F_{0} \oplus E_{1} \oplus d_{0} F_{0}$, with $E_{1} \simeq K_{1}$, we have shown that 1-loop contribution to the anomaly of an element in one of these subspaces belongs to the same subspace or to a subspace that stands to the right. Together with the last point of the previous list, this sums up the results for the elements of $H(\bar{s})$, i.e., for the obstructions and the bottoms contained in (4.13), (4.16) and (4.17) restricted to $r=0$.

In the same way, these results for all $r$ and $s$ follow from the fact that $\delta_{0}$ induces a well-defined differential (also called $\delta_{0}$ in the following) in the spaces $K_{r}$, anticommuting with $d_{r}, \delta_{0}: K_{r} \longrightarrow K_{r}$ with $\left\{\delta_{0}, d_{r}\right\}=0$.

Indeed, suppose this result to be true for $K_{0}, \ldots, K_{r-1}, d_{0}, \ldots, d_{r-1}$. An element $[a]_{r} \in K_{r}$ satisfies $\bar{s} a=0, d a+\bar{s} c_{1}=0, \ldots, d c_{r-1}+\bar{s} c_{r}=0$. This implies $s^{q} a \circ \Gamma^{\infty}=\hbar b \circ \Gamma^{\infty}$, $d a \circ \Gamma^{\infty}+s^{q} c_{1} \circ \Gamma^{\infty}=\hbar f_{1} \circ \Gamma^{\infty}, \ldots, d c_{r-1} \circ \Gamma^{\infty}+s^{q} c_{r} \circ \Gamma^{\infty}=\hbar f_{r} \circ \Gamma^{\infty}$. Applying $s^{q}$ gives to lowest order $\bar{s} b=0, d b+\bar{s} f_{1}=0, \ldots, d f_{r-1}+\bar{s} f_{r}=0$, so that $[b]_{r}$ is well defined. Suppose now that $[a]_{r}=d_{0}\left[g_{0}\right]_{0}+\ldots+d_{r-1}\left[g_{r-1}\right]_{r-1}$. Anticommutativity of $\delta_{0}$ with $d_{0}, \ldots, d_{r-1}$ then implies that $\delta_{0}[a]_{r}=0$. Hence, $\delta_{0}$ does not depend on the representative and is well defined in $K_{r}$. Finally, $d_{r} \delta_{0}[a]_{r}=d_{r}[b]_{r}=\left[d f_{r}\right]_{r}$, while $\delta_{0} d_{r}[a]_{r}=\delta_{0}\left[d c_{r}\right]_{r}$, and $s^{q}\left(d c_{r}\right) \circ \Gamma^{\infty}=s^{q} d\left(c_{r} \circ \Gamma^{\infty}+\hbar c^{\prime} \circ \Gamma^{\infty}\right)=-d\left(s^{q} c_{r} \circ \Gamma^{\infty}+\hbar s^{q} c^{\prime} \circ \Gamma^{\infty}\right)$, so that $\delta_{0}\left[d c_{r}\right]_{r}=$ $-\left[d\left(f_{r}+\bar{s} c^{\prime}\right)\right]_{r}=-d_{r} \delta_{0}[a]_{r}$.

The results (4.13), (4.16) and (4.17) can then be summarized by the statement that an anomaly in one of the subspaces of the decomposition (5.3) must belong to the same subspace or to one that stands to the right; furthermore, the part of the anomaly of an element of $F_{i}$ in $F_{i}$ is minus the part of the anomaly of the corresponding element of $d_{i} F_{i}$ in $d_{i} F_{i}$.

In order to recover the results for elements of $H(\bar{s} \mid d)$, we define $\Delta_{0}$ to be the equivalent of $\delta_{0}$ for modulo $d$ BRST cohomological classes, $\Delta_{0}[a]=[b]$, for $[a],[b] \in H(\bar{s} \mid d)$, where 
$\bar{s} a+d m=0, \bar{s} m+d u=0, s^{q} a \circ \Gamma^{\infty}+d\left(m \circ \Gamma^{\infty}\right)=\hbar b \circ \Gamma^{\infty}, s^{q} m \circ \Gamma^{\infty}+d\left(u \circ \Gamma^{\infty}\right)=\hbar n \circ \Gamma^{\infty}$. Indeed, the map is well defined because the consistency condition implies to lowest order $\bar{s} b+d n=0$, while if $a=\bar{s} c+d g$, we have $m=\bar{s} g+d u$, so that $s^{q} a \circ \Gamma^{\infty}+d m \circ \Gamma^{\infty}=\hbar b \circ \Gamma^{\infty}$ gives $s^{q}\left(s^{q} c \circ \Gamma^{\infty}+d g \circ \Gamma^{\infty}+\hbar f \circ \Gamma^{\infty}\right)+d\left(s^{q} g \circ \Gamma^{\infty}+d u \circ \Gamma^{\infty}+\hbar v \circ \Gamma^{\infty}\right)=\hbar b \circ \Gamma^{\infty}$, which implies $b=\bar{s} f+d v$ as it should.

The following properties are straightforward to check: $\left[\Delta_{0}, \mathcal{D}\right]=0, l \Delta_{0}=-\delta_{0} l, i_{0} \delta_{0}=$ $\Delta_{0} i_{0}$. One says (see e.g. [40], Chapter VIII.9) that $\left(\Delta_{0}, \delta_{0}\right)$ is a mapping of the exact couple $(H(\bar{s} \mid d), H(\bar{s}))$.

The previous result, that $\delta_{0}$ induces well defined maps in the spaces of the spectral sequence anticommuting with the differentials $d_{r}$, follows directly from the way the spectral sequence is associated to an exact couple. The relation between (4.5), (4.10) at $l=0$ and (4.16), (4.17) is summarized by $i_{0} \delta_{0}=\Delta_{0} i_{0}$; the relations between (4.5), (4.10) at different values of $l$ are summarized by $\left[\Delta_{0}, \mathcal{D}\right]=0$; finally, the relation between (4.10) at $l=r$ and (4.13) is summarized by $l \Delta_{0}=-\delta_{0} l$.

Note that in this case, we have furthermore the property that $\Delta_{0}$ is a differential, $\Delta_{0}^{2}=0$.

Remark: It follows from the above analysis that the relevant property of the differentials $d_{r}$ is $\left\{\delta_{0}, d_{r}\right\}=0$. This means that analogous results that constrain the anomalies to belong to particular subspaces of $H(\bar{s})$ or $H(\bar{s} \mid d)$ can be derived if one can find maps $\lambda_{0}: H(\bar{s}) \longrightarrow$ $H(\bar{s})$, respectively $\Lambda_{0}: H(\bar{s} \mid d) \longrightarrow H(\bar{s} \mid d)$ such that $\left[\delta_{0}, \lambda_{0}\right]=0$, respectively $\left[\Delta_{0}, \Lambda_{0}\right]=0$.

\section{ADLER-BARDEEN THEOREM REVISITED}

We now apply the ideas of the extended antifield formalism to standard Yang-Mills theory. In this case, it is sufficient for our purpose to couple the local BRST cohomology classes in ghost number 0 and ghost number 1, because this will be enough, under some assumptions stated explicitly below, to guarantee stability and to control the anomalies. The starting point action contains from the beginning a coupling to the Adler-Bardeen anomaly as in [18 20, with additional couplings to possibly higher dimensional gauge invariant operators, if one does not want to restrict oneself to the power counting renormalizable case [30]. More precicley, the starting point is the action

$$
\begin{array}{r}
S_{\rho}=\int d^{4} x\left[-\frac{1}{4 g^{2}} F_{I}^{\mu \nu} F_{\mu \nu}^{I}+L_{\text {matter }}^{\mathrm{kin}}\left(\psi^{i}, D_{\mu} \psi^{i}\right)\right] \\
+\int d^{4} x\left[-D_{\mu} C^{I} A_{I}^{* \mu}+C^{I} T_{I j}^{i} \psi^{j} \psi_{i}^{*}-\frac{1}{2} C^{I} C^{J} f_{J I}^{K} C_{K}^{*}\right] \\
+g^{i} \int d^{4} x \mathcal{O}_{i}+\mathcal{A} \rho,
\end{array}
$$

satisfying the master equation

$$
\frac{1}{2}\left(S_{\rho}, S_{\rho}\right)=0
$$

The Lagrangian $L_{\text {matter }}^{\mathrm{kin}}\left(\psi^{i}, D_{\mu} \psi^{i}\right)$ is the gauge invariant extension of the kinetic terms for the matter fields $\psi^{i}$. For simplicity, we assume the gauge group to be $S U(3)$. The $\mathcal{O}_{i}$ are gauge invariant local functions built out of the field strengths $F_{\mu \nu}^{I}$, the matter fields $\psi^{i}$ and their covariant derivatives such that the $\int d^{4} x \mathcal{O}_{i}$ (which can, but need not, be assumed to be 
power counting renormalizable) and $\int d^{4} x-\frac{1}{4 g^{2}} F_{I}^{\mu \nu} F_{\mu \nu}^{I}$ are linearily independent even when the gauge covariant equations of motions hold. Finally, $\mathcal{A}=\int \operatorname{Tr}\left[C d\left(A d A+\frac{1}{2} A^{3}\right)\right]$ is the Adler-Bardeen gauge anomaly, $g$ is the gauge coupling constant, $g^{i}$ are coupling constants for the other gauge invariant operators, while $\rho$ is a Grassmann odd coupling constant with ghost number -1 for the Adler-Bardeen anomaly. In this particular case, $\Delta_{c}=0$. This can be traced back to the fact that all representatives of the local BRST cohomological classes in ghost number 0 and 1 can be choosen to be independent of the antifields.

The gauge is fixed by introducing the cohomologically trivial non minimal sector consisting of the antighost $\bar{C}^{I}$ and the Lagrange multiplier $B^{I}$ and their antifields. One adds to the action (6.1) the term $\int d^{4} x \bar{C}_{I}^{*} B^{I}$ and chooses an appropriate gauge fixing fermion $\Psi$, which is used to generate an anticanonical transformation in the fields and antifields such that the propagators of the theory are well defined. The gauge fixing is irrelevant for the cohomological considerations below.

For the question of stability and anomalies, we have to analyze the cohomology $H^{0,4}\left(s_{\rho} \mid d\right)$ and $H^{1,4}\left(s_{\rho} \mid d\right)$ in the space of functions in the couplings $g, g^{i}, \rho$ with coefficients that are Lorentz invariant polynomials in the $d x^{\mu}$, the fields, the antifields and their derivatives.

In order to compute this cohomology, we decompose, as in [18, both the BRST differential $s_{\rho}$ and the local forms into parts independent of $\rho$ and parts linear in $\rho$. Explicitly, $s_{\rho}=s_{0}+s_{1}$, where $s$ is the standard BRST differential associated to the solution $S_{\rho=0}$ of the master equation, while $s_{1}=(\mathcal{A} \rho, \cdot)$. The $\rho$ independent part of the cocycle condition $s_{\rho} \omega(\rho)+d \eta(\rho)=0$ in form degree 4 gives (see e.g. [35] for a review) $\omega(0)=\alpha\left(g, g^{i}\right) d^{4} x F_{I}^{\mu \nu} F_{\mu \nu}^{I}+\alpha^{j}\left(g, g^{i}\right) d^{4} x \mathcal{O}_{i}+s()+d()$. This implies $\omega(\rho)=$ $\alpha\left(g, g^{i}\right) d^{4} x F_{I}^{\mu \nu} F_{\mu \nu}^{I}+\alpha^{j}\left(g, g^{i}\right) d^{4} x \mathcal{O}_{i}+\omega_{1}^{\prime} \rho+s_{\rho}()+d()$. Because $d^{4} x F_{I}^{\mu \nu} F_{\mu \nu}^{I}$ and $d^{4} x \mathcal{O}_{i}$ are also $s_{1}$ closed and $\rho^{2}=0$, the cocycle condition reduces to $s \omega_{1}^{\prime}+d()=0$, where the ghost number of $\omega_{1}^{\prime}$ is 1. It follows that $\omega_{1}^{\prime}=\lambda\left(g, g^{i}\right) \operatorname{Tr}\left[C d\left(A d A+\frac{1}{2} A^{3}\right)\right]+s()+d()$. Hence, the general solution of the consistency condition in the space of local functionals in ghost number 0 is given by

$$
\alpha\left(g, g^{i}\right) \frac{\partial S_{\rho}}{\partial g}+\alpha^{j}\left(g, g^{i}\right) \frac{\partial S_{\rho}}{\partial g^{i}}+\frac{\partial^{R} S_{\rho}}{\partial \rho} \rho \lambda\left(g, g^{i}\right)+\left(S_{\rho}, \Xi_{\rho}\right)
$$

for some local functional $\Xi_{\rho}$ in ghost number -1 . This implies that the theory is stable.

Similarily, in ghost number 1 , the $\rho$ independent part of the cohomology gives as only anomaly candidate the Adler-Bardeen anomaly. There could however be a $\rho$ linear non trivial contribution to the anomaly, because the cohomology of $s$ in form degree 4 and ghost number 2 is not necessarily empty (see [35], section 12.4), contrary to the claim in [18]. More precisely, to each $x^{\mu}$-independent, gauge and Lorentz invariant non trivial conserved current $j_{\Delta}=j_{\Delta}^{\mu} \epsilon_{\mu \nu_{1} \nu_{2} \nu_{3}} d x^{\nu_{1}} d x^{\nu_{2}} d x^{\nu_{3}}$, there corresponds the cohomological class $V_{\Delta}^{2,4}=j_{\Delta}\left[\operatorname{Tr} C^{3}\right]^{1}+$ antifield dependent terms in $H^{2, n}(s \mid d)$, with $s\left[\operatorname{Tr} C^{3}\right]^{1}+\operatorname{Tr} C^{3}=0$. (There could in principle be another type of antifield dependent cohomology classes in exceptional situations [35], which we exclude from the present considerations).

If there are such non trivial currents $j_{\Delta}$, we have to change our starting point and also couple the "anomaly for anomaly candidates" $V^{2,4}$ from the beginning with couplings in ghost number -2 . But then the cohomology of $s$ in ghost number 3 becomes relevant. There are plenty of such classes, for instance classes of the form $d^{4} x I \operatorname{Tr} C^{3}$, where $I$ are invariant functions built out of the field strengths, the matter fields and their covaraint 
derivatives. In this way, one is led to use the full extended antifield formalism as described in section II with all BRST cohomology classes in positive ghost number coupled from the beginning. In the case where the algebra of the non trivial symmetries associated to the currents $j_{\Delta}$ is non abelian, the operator $\Delta_{c}$ will be non vanishing at the classical level and involve in particular the structure constants of this algebra.

Another possibility is to try to show that the anomaly candidates $V_{\Delta}^{2,4}$ do not effectively arise in the theory, by using higher order cohomological restrictions: as in the proof of the absence of similiar instabilities in the presence of abelian factors for standard Yang-Mills theories (see [25], Appendix A) one couples with external fields gauge invariant functions that break the symmetries associated to the currents $j_{\Delta}$. In this way, one eliminates the currents $j_{\Delta}$ and the associated anomaly for anomaly candidates $V_{\Delta}^{2,4}$ from the extended theory. At the end of the computations, the external fields can be put to zero.

Because this discussion is not central to the argument below, we will simply assume here that the observables $\mathcal{O}_{i}$ are such that there are no non trivial currents $j_{\Delta}$ and thus no anomaly for anomaly candidates $V_{\Delta}^{2,4}$ in the theory. The general solution of the consistency condition in ghost number 1 is then given by

$$
\frac{\partial S_{\rho}}{\partial \rho} \sigma\left(g, g^{i}\right)+\left(S_{\rho}, \Sigma_{\rho}\right)
$$

for some local functional $\Sigma_{\rho}$ in ghost number 0 .

By standard arguments, using in addition the same reasoning as in section Пए follows from (6.3) and (6.4) that the model is renormalizable and that the renormalized generating functional for 1 particle irreducible vertex functions $\Gamma_{\rho}$ satisfies

$$
\frac{1}{2}\left(\Gamma_{\rho}, \Gamma_{\rho}\right)+\frac{\partial^{R} \Gamma_{\rho}}{\partial \rho} \hbar \sigma\left(g, g^{i}\right)=0
$$

where $\sigma\left(g, g^{i}\right)$ is a formal power series in $\hbar$. Hence, in this case $\Delta^{\infty}=\frac{\partial^{R}}{\partial \rho} \hbar \sigma\left(g, g^{i}\right)$ and the quantum BRST differential is $s^{q}=\left(\Gamma_{\rho}, \cdot\right)-\hbar \sigma\left(g, g^{i}\right) \partial^{L} \partial \rho$. (It can be shown along standard lines that the coefficient $\sigma\left(g, g^{i}\right)$ does not depend on the parameters of the gauge fixing.)

Let us now investigate the renormalization of the operators $d^{4} x F_{I}^{\mu \nu} F_{\mu \nu}^{I}$ and $d^{4} x \mathcal{O}_{i}$. According to the classification in section [II], they are both of the type $e_{\alpha_{0}}^{0}$, because they are non trivial bottoms in maximal form degree 4, while the Adler-Bardeen anomaly $\operatorname{Tr}\left[C d\left(A d A+\frac{1}{2} A^{3}\right)\right]$ is of the type $e_{\alpha_{4}}^{4}$, as it descends to the non trivial bottom $\operatorname{Tr} C^{5}$. Because there is no $e_{\alpha_{0}}^{0}$ in ghost number 1 (and form degree 4) and no $h_{i_{r}}^{r}$ in form degree 3 and ghost number 1, equation (4.5) implies that the lowest order contribution to the anomaly in the renormalization of $d^{4} x F_{I}^{\mu \nu} F_{\mu \nu}^{I}$ and of $d^{4} x \mathcal{O}_{i}$ is $s_{\rho}$ exact and can thus be absorbed through a BRST breaking counterterm added to these operators. This reasoning can be pushed to all orders, with the result that one can achieve, through the addition of suitable counterterms,

$$
\begin{array}{r}
s^{q}\left[d^{4} x F_{I}^{\mu \nu} F_{\mu \nu}^{I} \circ \Gamma_{\rho}\right]=0, \\
s^{q}\left[d^{4} x \mathcal{O}_{i} \circ \Gamma_{\rho}\right]=0 .
\end{array}
$$

If we now apply $-\frac{g}{2} \frac{\partial}{\partial g}$, respectively $\frac{\partial}{\partial g^{j}}$ to 6.5 , we get on the one hand, 


$$
\begin{aligned}
s^{q}\left[\int d^{4} x-\frac{1}{4 g^{2}} F_{I}^{\mu \nu} F_{\mu \nu}^{I} \circ \Gamma_{\rho}\right]+\frac{\partial^{R} \Gamma_{\rho}}{\partial \rho} \hbar\left[-\frac{g}{2} \frac{\partial \sigma\left(g, g^{i}\right)}{\partial g}\right] & =0, \\
s^{q}\left[\int d^{4} x \mathcal{O}_{i} \circ \Gamma_{\rho}\right]+\frac{\partial^{R} \Gamma_{\rho}}{\partial \rho} \hbar\left[\frac{\partial \sigma\left(g, g^{i}\right)}{\partial g^{j}}\right] & =0 .
\end{aligned}
$$

Comparing on the other hand with the integrated versions of (6.6) and (6.7), we deduce that

$$
\frac{\partial \sigma\left(g, g^{i}\right)}{\partial g}=0, \frac{\partial \sigma\left(g, g^{i}\right)}{\partial g^{j}}=0,
$$

which is an expression of the Adler-Bardeen theorem (see e.g. section 6.3.2 of [29]).

\section{CONCLUSION AND OUTLOOK}

In the completely extended antifield formalism, where in particular potential anomaly candidates are coupled to the starting point action with independent couplings, the whole program of algebraic renormalization can be extended to the case of theories with gauge anomalies. For instance, it is possible to write the Callan-Symanzik equation to all orders as a functional differential equation as in [31], by using $\Delta^{\infty}$ instead of $\Delta_{c}$. The $\beta$ functions can then be considered as anomaly coefficients in the renormalization of the cohomology class corresponding to the generator of dilatations. Vanishing results for $\beta$ functions associated to couplings of cohomological classes with a non trivial descent can then be obtained as a particular case of the discussion of section IV.

The discussion in section $\mathbb{I V}$ on the length of descents and lifts of BRST cohomological classes and their anomalies does not rely on the use of the extended antifield formalism. It can be done along the same lines in the standard set-up as long as one assumes the quantum theory to be anomaly free and stable, so that the standard Zinn-Justin equation $\frac{1}{2}(\Gamma, \Gamma)=0$

holds. A virtue of the completely extended formalism is that it allows in principle to treat the general case.

\section{ACKNOWLEDGMENTS}

This work has been partly supported by the "Actions de Recherche Concertées" of the "Direction de la Recherche Scientifique - Communauté Française de Belgique", by IISN Belgium (convention 4.4505.86). The author wants to thank F. Brandt and M. Henneaux for useful discussions. 


\section{REFERENCES}

* Scientific Research Worker of the "Fonds National Belge de la Recherche Scientifique".

[1] J. Wess and B. Zumino, "Consequences Of Anomalous Ward Identities," Phys. Lett. B37 (1971) 95.

[2] C. Becchi, A. Rouet and R. Stora, "The Abelian Higgs-Kibble Model. Unitarity Of The S Operator," Phys. Lett. B52 (1974) 344.

[3] C. Becchi, A. Rouet and R. Stora, "Renormalization Of The Abelian Higgs-Kibble Model," Commun. Math. Phys. 42 (1975) 127.

[4] C. Becchi, A. Rouet and R. Stora, "Renormalization Of Gauge Theories," Annals Phys. 98 (1976) 287.

[5] B.L. Voronov and I.V. Tyutin, "Renormalization Of Two-Dimensional Chiral Theories. (In Russian)," Yad. Fiz. 33 (1981) 1137.

[6] C. Becchi, A. Blasi, G. Bonneau, R. Collina and F. Delduc, "Renormalizability And Infrared Finiteness Of Nonlinear Sigma Models: A Regularization Independent Analysis For Compact Coset Spaces," Commun. Math. Phys. 120 (1988) 121.

[7] I.A. Batalin and G.A. Vilkovisky, "Gauge Algebra And Quantization," Phys. Lett. 102B (1981) 27.

[8] I.A. Batalin and G.A. Vilkovisky, "Quantization Of Gauge Theories With Linearly Dependent Generators," Phys. Rev. D28 (1983) 2567.

[9] P.S. Howe, U. Lindström and P. White, "Anomalies And Renormalization In The BRSTBV Framework," Phys. Lett. B246 (1990) 430.

[10] W. Troost, P. van Nieuwenhuizen and A. Van Proeyen, "Anomalies And The BatalinVilkovisky Lagrangian Formalism," Nucl. Phys. B333 (1990) 727.

[11] F. Brandt, M. Henneaux and A. Wilch, "Extended antifield formalism," Nucl. Phys. B510 (1998) 640 [hep-th/9705007.

[12] J. Dixon and M. Ramon Medrano, "Anomalies of higher-dimension composite fields," Phys. Rev. D22 (1980) 429.

[13] R. Stora, "Continuum Gauge Theories," Lectures given at Summer Inst. for Theoretical Physics, Cargèse, France, Jul 12-31, 1976, published in New Developments in Quantum Field Theory and Statistical Mechanics, eds. M. Lévy and P. Mitter (Plenum Press, 1977).

[14] R. Stora, "Algebraic Structure And Topological Origin Of Anomalies," Seminar given at Cargèse Summer Inst. Sep 1-15, 1983, published in Progress in Gauge Field Theory, eds. 't Hooft et al. (Plenum Press, 1984).

[15] B. Zumino, "Chiral Anomalies And Differential Geometry," Lectures given at Les Houches Summer School on Theoretical Physics, Les Houches, France, Aug 8 - Sep 2, 1983, published in Relativity, Groups and Topology II, eds. B.S. DeWitt and R. Stora (North Holland, Amsterdam 1984).

[16] B. Zumino, Y. Wu and A. Zee, "Chiral Anomalies, Higher Dimensions, And Differential Geometry," Nucl. Phys. B239 (1984) 477.

[17] M. Dubois-Violette, M. Talon and C.M. Viallet, "BRS Algebras: Analysis Of The Consistency Equations In Gauge Theory," Commun. Math. Phys. 102 (1985) 105.

[18] G. Costa, J. Julve, T. Marinucci and M. Tonin, "Nonabelian Gauge Theories And Triangle Anomalies," Nuovo Cim. 38A (1977) 373. 
[19] S. Aoyama and M. Tonin, "The Dimensional Regularization Of Chiral Gauge Theories And Generalized Slavnov-Taylor Identities," Nucl. Phys. B179 (1981) 293.

[20] M. Tonin, "Dimensional regularization and anomalies in chiral gauge theories," Nucl. Phys. Proc. Suppl. 29BC (1992) 137.

[21] W.A. Bardeen, "Renormalization of Yang-Mills fields and application to particle physics," C.N.R.S. (Marseille) report 72/P 470 (1972) 29 ; Proceedings of the Sixteenth International conference on High Energy Physics, Fermilab (1972) vol. 2, 295.

[22] G. Bandelloni, C. Becchi, A. Blasi and R. Collina, "On The Cancellation Of Hard Anomalies In Gauge Field Models: A Regularization Independent Proof," Commun. Math. Phys. 72 (1980) 239.

[23] O. Piguet and S. P. Sorella, "On the finiteness of the BRS modulo d cocycles," Nucl. Phys. B381 (1992) 373 [hep-th/9302122].

[24] O. Piguet and S. P. Sorella, "Adler-Bardeen theorem and vanishing of the gauge beta function," Nucl. Phys. B395 (1993) 661 hep-th/9302123.

[25] G. Barnich, "On the quantum Batalin-Vilkovisky formalism and the renormalization of non linear symmetries," JHEP 9903 (1999) 010 [hep-th/9807210.

[26] G. Barnich, "Higher order cohomological restrictions on anomalies and counterterms," Phys. Lett. B419 (1998) 211 [hep-th/9710162].

[27] M. Henneaux and C. Teitelboim, "Quantization of gauge systems," Princeton, USA: Univ. Pr. (1992) $520 \mathrm{p}$.

[28] G. Bonneau, "Some Fundamental But Elementary Facts On Renormalization And Regularization: A Critical Review Of The Eighties," Int. J. Mod. Phys. A5 (1990) 3831.

[29] O. Piguet and S.P. Sorella, "Algebraic renormalization: Perturbative renormalization, symmetries and anomalies," Berlin, Germany: Springer (1995) 134 p. (Lecture notes in physics: m28).

[30] J. Gomis and S. Weinberg, "Are Nonrenormalizable Gauge Theories Renormalizable?," Nucl. Phys. B469 (1996) 473 [hep-th/9510087].

[31] G. Barnich, "A general non renormalization theorem in the extended antifield formalism," JHEP 9812 (1998) 003 hep-th/9805030.

[32] J.H. Lowenstein, "Differential Vertex Operations In Lagrangian Field Theory," Commun. Math. Phys. 24 (1971) 1.

[33] Y.P. Lam, "Perturbation Lagrangian Theory For Scalar Fields: Ward-Takahasi Identity And Current Algebra," Phys. Rev. D6 (1972) 2145.

[34] Y.P. Lam, "Equivalence Theorem On Bogoliubov-Parasiuk-Hepp-Zimmermann Renormalized Lagrangian Field Theories," Phys. Rev. D7 (1973) 2943.

[35] G. Barnich, F. Brandt and M. Henneaux, "Local BRST Cohomology in Gauge Theories," Phys. Rept., to appear.

[36] M. Talon, "Algebra Of Anomalies," PAR-LPTHE-85/37, Presented at Cargèse Summer School, Cargèse, France, Jul 15-31, 1985.

[37] F. Brandt, N. Dragon and M. Kreuzer, "All solutions of the Wess-Zumino consistency conditions," in Geometry and Theoretical Physics, pp. 243-266, (Springer-Verlag, Berlin 1991).

[38] M. Henneaux and B. Knaepen, "The Wess-Zumino consistency condition for p-form gauge theories," Nucl. Phys. B548 (1999) 491 hep-th/9812140.

[39] C. Lucchesi, O. Piguet and K. Sibold, "The Adler-Bardeen Theorem For The Axial 
U(1) Anomaly In A General Nonabelian Gauge Theory," Int. J. Mod. Phys. A2 (1987) 385.

[40] S.-T. Hu, "Homotopy Theory," Academic Press (New York 1959). 